\title{
(2) OPEN ACCESS \\ Impact on the fitness of N95 masks with extended use/limited reuse and dry heat decontamination
}

\author{
Mengyi Zha 이 , ${ }^{1}$ Jude Alsarraj, ${ }^{1}$ Brandon Bunch, ${ }^{2}$ David Venzon ${ }^{3}$
}

\begin{abstract}
Medical Department, Columbia Basin Health Association, Othello, Washington, USA ${ }^{2}$ Chemistry Department, Washington State University, Pullman, Washington, USA ${ }^{3}$ Biostatistics and Data Management Section, National Cancer Institute, National Institutes of Health, Bethesda, Maryland, USA
\end{abstract}

Correspondence to Dr Mengyi Zha, Medical Department, Columbia Basin Health Association, Othello, WA 99344, USA: zedzha@gmail.com

Accepted 27 July 2021
Check for updates

(C) American Federation for Medical Research 2021. Re-use permitted under CC BY-NC. No commercial re-use. Published by BMJ.

To cite: Zha M,
Alsarraj J, Bunch B, et al.
J Investig Med Epub
ahead of print: [please
include Day Month Year].
doi:10.1136/jim-2021-
001908

\begin{abstract}
Substandard use of N95 masks, sometimes combined with dry heat decontamination, lacks safety data. We evaluated the impact of these practices on the fitness of N95 masks. This is a non-human subject research conducted from July to October 2020. 155 masks were used by 12 healthcare workers during 10-hour shifts. Masks were collected at the end of the shift and if the number of donnings/doffings was less than five ('modified extended use', ME) or whenever this number reached five ('limited reuse', LR), per the recommendation of the Centers for Disease Control and Prevention. Masks that passed an Occupational Safety and Health Administration qualitative fit test underwent a cycle $\left(30 \mathrm{~min}, 75^{\circ} \mathrm{C}\right)$ of dry heat decontamination. After use, $84 \%$ (95\% Cl $77 \%$ to $90 \%$ ) of the masks fit the users, $85 \%$ (95\% Cl $73 \%$ to $93 \%)$ in ME and $83 \%(95 \% \mathrm{Cl} 73 \%$ to $90 \%)$ in LR. After dry heat, $86 \%$ of the fitted masks ( $95 \% \mathrm{Cl} 78 \%$ to $91 \%$ ) still fit, $93 \%(95 \% \mathrm{Cl} 80 \%$ to $98 \%)$ in $\mathrm{ME}$ and $82 \%(95 \% \mathrm{Cl}$ $70 \%$ to $89 \%$ ) in LR. If a fit test was not done before decontamination, $72 \%$ (95\% Cl $64 \%$ to $79 \%$ ) of the masks would fit, $79 \%$ (95\% Cl $66 \%$ to $88 \%$ ) in ME and $68 \%$ (95\% Cl $57 \%$ to $77 \%$ ) in LR. Common substandard use preserves fitness of N95 masks up to $85 \%$. One cycle of dry heat decontamination preserves fitness of N95 masks up to $93 \%$ when donned/ doffed less than five times and fitness is ensured before decontamination. If a fit test is not performed beforehand, dry heat decontamination cannot preserve the fitness of used N95 masks above 80\%.
\end{abstract}

\section{INTRODUCTION}

All US Food and Drug Administration (FDA)approved N95 respirator masks are cleared for single-use. This means they should be replaced after each patient encounter. ${ }^{1}$ However, the ongoing COVID-19 pandemic has left these masks in short supply for healthcare workers, necessitating the need for reuse. ${ }^{23}$ Extended use refers to the practice of wearing the same N95 mask for multiple patient encounters without donning/doffing, while limited reuse is defined as limiting encounter-driven donning/doffing of a single mask to less than five times. ${ }^{1}$

Although not recommended by major N95 manufacturers such as $3 \mathrm{M},{ }^{4}$ substandard use of

\section{Significance of this study}

What is already known about this subject?

- All Food and Drug Administration (FDA)approved N95 respirator masks are cleared for single-use, but in the current COVID-19 pandemic substandard use is common.

- The Centers for Disease Control and Prevention recommends that donning/ doffing an N95 mask during reuse should be limited to less than five times, according to data from previous studies.

- Prior studies assessing the safety of N95 substandard use were either performed in a non-clinical setting or were cross-sectional.

- Studies on heat decontamination methods of N95 masks were done using new or unused masks or in laboratory settings, and no dry heat N95 decontamination unit has been granted emergency use authorization by the FDA due to lack of data and standardization.

What are the new findings?

- Limited reuse and modified extended use, when carefully done, can preserve fitness of N95 masks up to $85 \%$.

- Dry heat decontamination method after substandard use can preserve fitness of N95 masks up to $93 \%$ if the number of donnings/doffings is less than five and a fit test is performed before decontamination.

- If a fit test is not performed after use and before decontamination, which is the most common scenario, fitness can only be preserved to up to $79 \%$ in masks that have been donned/doffed less than five times.

N95 masks is frequently combined with decontamination methods such as dry heat. Some recent research has studied the effects of either extended use, limited reuse or heat decontamination. Limited literature exists that quantifies the combined impact of both, especially in a practical setting. ${ }^{5-10}$

\section{MATERIALS AND METHODS}

This is a single-site, non-human subject research conducted from July to October 2020 where 


\section{Significance of this study}

How might these results change the focus of research or clinical practice?

- Although common substandard use of N95 masks as part of pandemic planning is suboptimal in terms of safety, careful enforcement of self-checks, following the five donnings/doffings rule, and limiting the duration of use can make substandard use relatively safe for healthcare workers.

- When using dry heat decontamination, care should be taken to limit the number of donnings/doffings to less than five and fitness should be ensured before decontamination.

the study subjects were N95 masks. One type of mask was used for this study, the 3M 9211 mask. The study site was a dental clinic that is part of a large rural primary care clinic located in eastern Washington state.

Among the staff fitted for the study masks, those who did not work on regular 10-hour shifts, were unwilling to consent to the study, tested positive for COVID-19, or were pregnant were excluded. A total of 12 volunteers met the criteria for the study and participated throughout. All volunteers passed the Occupational Safety and Health Administration-mandated N95 qualitative fit test with saccharin aerosol as a testing agent (the fit test) for the study mask.

At the start of their 10-hour shifts, each volunteer obtained a new mask. The mask was worn for at most one shift. The 3M Particulate Respirator N95 User Instructions were followed. ${ }^{11}$ A user check according to the instructions was done before each use to ensure the masks were in good operating condition. At any time, a mask was discarded if it did not pass the user check due to reasons such as any obvious hardware failure, deformity, air leakage, or if the mask was completely soiled or wet. Participants agreed not to wear heavy make-up, keep their face clean-shaven, and to not alter or wash the masks while using them. Participants were asked to count and keep track of their donning/doffingby writing them down on collection bags, which were breathable and made of paper. Extraordinary use beyond routine patient care was discouraged. Additionally, since the study masks were equipped with valves, all participants used a surgical mask on top of the N95 mask to protect the patients.

A worn mask meeting the above criteria was collected after the 10-hour shift and if the number of donnings/doffings was less than five ('modified extended use', ME group) or if at any time within a single shift thenumber of donnings/ doffings reached exactly five ('limited reuse', LR group). In other words, ME masks were used for 10 hours with less than five donnings/doffings, and LR masks were used for less than 10 hours with exactly five donnings/doffings. A limit of five for donning/doffing was used per the Centers for Disease Control and Prevention (CDC) recommendation. ${ }^{12}{ }^{13}$ Each individual mask was placed in a breathable paper bag with a de-identified letter code assigned to each wearer. Masks in the LR and ME groups were collected in separate containers away from contaminated areas at the

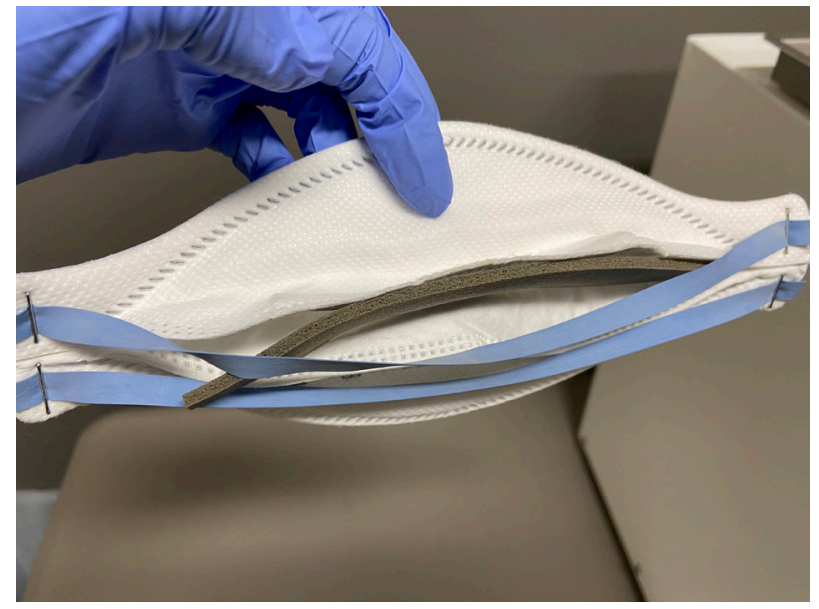

Figure 1 An example of study N95 mask with adhesive failure, resulting in detachment of the soft foam nose seal from the mask

end of each work day. The storage room's humidity was less than $50 \%$ relative humidity and the temperature was about $20^{\circ} \mathrm{C}$.

A fit test was performed on all collected masks after allowing the masks to air out for 72 hours, known as the time-decontamination method. ${ }^{6}$ All fitted masks then underwent a full cycle $\left(30 \mathrm{~min}, 75^{\circ} \mathrm{C}\right)$ of high velocity hot air (dry heat) decontamination using a commercial N95 sterilizer unit. This setting was chosen according to the manufacturer's user instruction and investigation. This temperature setting does not exceed the temperature limit $3 \mathrm{M}$ recommends against. ${ }^{4}$ All masks were carefully inspected after decontamination to evaluate for integrity following $3 \mathrm{M}$ instructions. The only type of visible damage that occurred was to the soft foam nose seal, connected to the respirator via adhesive, resulting in gaps or detachment from the mask (figure 1). Since previous studies showed that this type of defect might not interfere with fitness, all decontaminated masks were fit-tested. ${ }^{14}$

There are two primary outcomes in this study: the percentage of masks that fit after ME and LR and the percentage of masks that fit after one round of dry heat.

To assess the percentage of masks that passed the fit test after substandard use and after dry heat decontamination, 95\% CIs were used. All statistical calculations were done using the GraphPad QuickCalcs website (https://www. graphpad.com/quickcalcs/, accessed May 2021).

\section{RESULTS}

\section{Mask retention after use}

The study used 155 new masks. After use, 126 or $81 \%$ (95\% CI $74 \%$ to $87 \%$ ) of the total intact masks were retained, 48 in the ME group and 78 in the LR group. Notably, 29 masks were discarded during the 10-hour shifts due to not passing the user check as mentioned above. It is important to mention here that if the five donnings/doffings rule was followed and masks in the LR group were discarded as per the CDC recommendation, ${ }^{12}$ only 48 or $31 \%$ (95\% CI $24 \%$ to $39 \%$ ) of the masks would be retained after use. 
Table 1 Mask fitness after use and after dry heat decontamination

\begin{tabular}{|c|c|c|c|c|c|c|}
\hline & \multicolumn{2}{|c|}{ All masks } & \multicolumn{2}{|l|}{ ME } & \multicolumn{2}{|l|}{ LR } \\
\hline & $\%$ & $95 \% \mathrm{Cl}$ & $\%$ & $95 \% \mathrm{Cl}$ & $\%$ & $95 \% \mathrm{Cl}$ \\
\hline Fitness after use & 84 & 77 to 90 & 85 & 73 to 93 & 83 & 73 to 90 \\
\hline Fitness after dry heat: fit test done prior to heat & 86 & 78 to 91 & 93 & 80 to 98 & 82 & 70 to 89 \\
\hline Fitness after dry heat: no fit test done prior to heat & 72 & 64 to 79 & 79 & 66 to 88 & 68 & 57 to 77 \\
\hline
\end{tabular}

$L R$, limited reuse; $M E$, modified extended use.

\section{Fitness after use}

After ME and LR, 106 or 84\% (95\% CI 77\% to 90\%) of the used masks passed the fit test, 41 or $85 \%$ (95\% CI $73 \%$ to 93\%) passed in the ME group and 65 or $83 \%$ (95\% CI $73 \%$ to $90 \%$ ) passed in the LR group.

\section{Fitness after dry heat decontamination}

All 106 fitted masks after substandard use underwent one cycle of decontamination with dry heat. Of the masks, 91 or $86 \%$ (95\% CI 78\% to $91 \%$ ) passed the fit test after dry heat, 38 or $93 \%$ (95\% CI $80 \%$ to $98 \%$ ) passed in the ME group and 53 or $82 \%(95 \%$ CI $70 \%$ to $89 \%)$ passed in the LR group. In clinical practice, a fit test is not usually done after use before decontamination. If no fit test was done at this juncture and all 126 retained masks after use were decontaminated, then 91 or $72 \%$ (95\% CI 64\% to 79\%) would pass the fit test, 38 or $79 \%$ (95\% CI $66 \%$ to $88 \%$ ) would pass in the ME group and 53 or $68 \%$ (95\% CI 57\% to 77\%) would pass in the LR group (table 1). This is assuming that masks that failed the fit test before decontamination would still fail after decontamination.

\section{DISCUSSION}

Prior studies of N95 substandard use were either performed in a non-clinical setting ${ }^{13}$ or were cross-sectional. ${ }^{589}$ Additionally, studies done on heat decontamination methods of N95 masks were performed using new or unused masks in laboratory settings. ${ }^{71015-19}$ Our study is the only one so far that cohorts the study subjects in a clinical setting, with a combination of dry heat decontamination.

The Bergman et al study is the quintessential piece that influenced the CDC's five donnings/doffings recommendation. Bergman et $a l^{13}$ considered more than 5\% mask failure rate or less than $95 \%$ fitness rate to be unacceptable. If this standard is used, then according to our findings neither ME or LR, even with strict user self-check to eliminate obviously unfit masks, is considered safe use.

During a pandemic where personal protective equipment resource is scarce, the tolerance for fitness failure may be higher. Our results indicate that if $85 \%$ fitness rate standard is considered acceptable, then ME and LR can be considered largely safe when carefully practiced (84\% fitness rate, $95 \%$ CI $77 \%$ to $90 \%$ ), with ME safer (85\% fitness rate, $95 \%$ CI $73 \%$ to $93 \%)$ than LR (83\% fitness rate, 95\% CI $73 \%$ to $90 \%)$. In any case, our data speak to the importance of enforcing safety rules for substandard use by healthcare organizations, which means emphasizing user checks of masks to eliminate masks that have any obvious fitness failure, limiting donning/doffing to less than five times, and ideally limiting the duration of substandard use to less than 10 hours.
Similarly, if an organization can accept less than 95\% fitness rate for N95 masks after decontamination, dry heat would be an easy and low labor-intensive decontamination method for masks that have been donned/doffed less than five times and passed the fit test before decontamination (93\% fitness rate, 95\% CI 80\% to 98\%). However, our protocol ensured mask fitness before decontamination and masks that failed the fit test during this step did not make it to the decontamination unit. Although this helps improve safety, it can be impractical. If this was not done, then after one cycle of dry heat, the ME and LR groups would both fall below $80 \%$ fitness rate, although ME masks were still safer for use (79\% fitness rate, 95\% CI 66\% to $88 \%$ ) compared with LR masks (68\% fitness rate, 95\% CI $57 \%$ to $77 \%$ ).

Additionally, the dry heat decontamination method, although commonly used due to its low labor intensity and cost, lacks standardization data. In fact, so far, no dry heat decontamination sterilizer has obtained emergency use authorization (EUA) through the FDA. ${ }^{20} 3 \mathrm{M}$ tested several types of unused masks using the bioburden reduction systems that have been issued an enforcement policy by the FDA for 10 cycles, each cycle under $70^{\circ} \mathrm{C}$ dry heat for $60 \mathrm{~min}$. The results suggested safety in terms of filtration efficiency and fitness after 10 cycles. ${ }^{4}$ The enforcement policy for the bioburden reduction systems states that either $70^{\circ} \mathrm{C}$ for $60 \mathrm{~min}$ or $75^{\circ} \mathrm{C}$ for $30 \mathrm{~min}$ can be used and still maintain respirator integrity. ${ }^{21}$ Although we used a different commercial sterilizer unit, our sterilizing condition is the same as mentioned above $\left(75^{\circ} \mathrm{C}\right.$ for $\left.30 \mathrm{~min}\right)$. In contrast to the above existing information, our data speak to the limited utility and safety profile of dry heat decontamination beyond one cycle in real practice, where masks have been used before decontamination.

We want to point out that $85 \%$ respirator fitness rate after use means, clinically, that at the end of use, among every 100 staff, 15 might be exposed to a respiratory pathogen such as SARS-CoV-2. Acceptance of this substandard use should be carefully considered by the ethics committee of an organization. Ultimately, increasing production of personal protective equipment is the real solution to this concern.

\section{Limitations}

Our study only included one type of masks, the 3M 9211 mask, which previously was a commonly used foldable mask with a valve and has since been discontinued by $3 \mathrm{M}^{22}$ According to a $3 \mathrm{M}$ sales representative, the reason for the discontinuation of this mask is part of their ongoing efforts to focus production on the few lines of masks that are of higher demand. There are, in general, two shapes of N95 respirators: foldable and dome-shaped. The mask 
used in our study can likely be generalized to other foldable types of masksused in a setting similar to our study. This is because we theorize that the main reasons for reduced fitness are loss of elasticity of rubber bands due to donning/ doffing and adhesive failure/melting due to heat, not due to filtration failure, which is supported by previous studies. ${ }^{23}$ However, future studies should include non-valve types and dome-shaped N95 masks.

The CDC defines extended use as 'wearing the same N95 respirator for repeated close contact encounters with several patients, without removing the respirator between patient encounters'. ${ }^{12}$ In actual practice, this is not easily sustained for more than a few hours since the user will need to take breaks for hydration, food, mask intolerance, etc, especially in the outpatient setting. In the intensive care units or COVID-19-specific units, strict extended use is likely more easily enforced. Our protocol for ME use is more commonly adopted by organizations in outpatient settings. Studies examining the safety of extended use defined by the CDC are needed.

The commercial dry heat sterilizer used in this study has not been issued an enforcement policy or EUA by the FDA. As mentioned above, no dry heat sterilizer unit has obtained EUA through the FDA. Furthermore, although our study mask closely resembles several types of masks tested by $3 \mathrm{M}$ for dry heat decontamination method, it was not one of the tested types.

We performed a fit test after substandard use and before dry heat decontamination, and only those masks that passed the fit test went into the sterilizer for decontamination. This step is not usually performed in real practice. To deduce the fitness rate of $\mathrm{N} 95$ masks without a fit test at this juncture, we assumed that those masks that did not pass the fit test before decontamination would not pass after decontamination. While it is reasonable to assume this, we cannot say for sure that dry heat only negatively impacts the fitness of N95 masks.

Lastly, although our findings provide some safety data regarding reuse and the dry heat decontamination method, we do not provide data on how long the fitness lasts during further reuse. Longer-term studies that allow repeated reuse and use after dry heat decontamination cycles are needed for further assessment.

\section{CONCLUSIONS}

This non-human subject cohort study found that substandard use of N95 masks such as modified extended use and limited reuse, although these cannot preserve fitness of masks above $95 \%$, can achieve fitness rate of $85 \%$. Our findings also confirm that it is important to follow strict user checks recommended by mask manufacturers, follow the five donnings/doffings rule recommended by the CDC, and limit duration of use ideally to less than 10 hours.

Similarly, the dry heat decontamination method after substandard use, although it cannot preserve fitness of masks above $95 \%$, can achieve fitness rate of up to $93 \%$ if the number of donnings/doffings is less than five and a fit test is performed before decontamination. In real practice, where a fit test is usually not performed before decontamination, dry heat decontamination can only preserve fitness of up to $79 \%$ in masks that have been donned/doffed less than five times. This finding suggests that when using dry heat decontamination, it is important to limit the number of donnings/doffings to less than five and ensure that only fitted masks would undergo this method of decontamination for reuse. Dry heat decontamination most likely has limited utility beyond one cycle in real practice.

As many healthcare organizations are practicing substandard use of N95 masks due to shortage during the COVID-19 pandemic, our findings provide some safety considerations. Since dry heat decontamination is one of the most common methods used to preserve N95 masks, our findings can help organizations select masks that are safer to undergo this method of decontamination.

Acknowledgements We would like to thank Ji Choi, DDS, Tawni Solberg, MHA, and Kristen Stevens, RN, for their technical and intellectual support, and Chi-Ping Day, PhD, for his intellectual discussions. We would like to thank all of the volunteers who participated in this study. We would like to thank all members of the executive team at Columbia Basin Health Association for helpful discussions and financial support.

Contributors MZ: designing, planning, data collecting, data processing, manuscript writing, editing, approval of final draft. JA: designing, planning, data processing, manuscript writing, editing, approval of final draft. BB: planning, data collecting, editing, approval of final draft, conference presentation. DV: processing, analysis, interpretation of data, revising drafts, approval of final version of draft.

Funding The authors have not declared a specific grant for this research from any funding agency in the public, commercial or not-for-profit sectors.

Competing interests None declared.

Patient consent for publication Not required.

Ethics approval Our study was determined to be non-human subject research and therefore exempt by the Advarra IRB review board.

Provenance and peer review Not commissioned; externally peer reviewed.

Data availability statement All data relevant to the study are included in the article or uploaded as supplemental information. All data were deidentified. Data storage and processing were done using Excel documents and saved in a secure folder in Columbia Basin Health Association company folders, with access only granted to MZ and JA.

Open access This is an open access article distributed in accordance with the Creative Commons Attribution Non Commercial (CC BY-NC 4.0) license, which permits others to distribute, remix, adapt, build upon this work noncommercially, and license their derivative works on different terms, provided the original work is properly cited, an indication of whether changes were made, and the use is non-commercial. See: http://creativecommons.org/ licenses/by-nc/4.0/.

ORCID iD

Mengyi Zha http://orcid.org/0000-0003-3034-1255

\section{REFERENCES}

1 U.S. Food and Drug Administration. N95 respirators, surgical masks, and face masks, 2020. Available: https://www.fda.gov/medical-devices/personalprotective-equipment-infection-control/n95-respirators-surgical-masks-andface-masks

2 Occupational Safety and Health Administration. Enforcement guidance for respiratory protection and the N95 shortage due to the coronavirus disease 2019 (COVID-19) pandemic, 2020. Available: https://www.osha.gov/memos/ 2020-04-03/enforcement-guidance-respiratory-protection-and-n95-shortagedue-coronavirus

3 Fisher EM, Shaffer RE. Considerations for recommending extended use and limited reuse of filtering facepiece respirators in health care settings. J Occup Environ Hyg 2014;11:D115-28.

4 Decontamination of 3M Filtering Facepiece Respirators, such as N95 Respirators, in the United States - Considerations [press release]. multimedia. 3m.com 2021.

5 Degesys NF, Wang RC, Kwan E, et al. Correlation between N95 extended use and reuse and fit failure in an emergency department. JAMA 2020;324:94-6. 
6 Su-Velez BM, Maxim T, Long JL, et al. Decontamination methods for reuse of filtering Facepiece respirators. JAMA Otolaryngol Head Neck Surg 2020;146:734.

7 Liao L, Xiao W, Zhao M, et al. Can N95 respirators be reused after disinfection? how many times? ACS Nano 2020:14:6348-56.

8 Check R, Kelly B, Rivard L, et al. 7 failure rates during reuse of disposable N95 masks in clinical practice in the emergency department. Ann Emerg Med 2020;76:S3-4.

9 Maranhao B, Scott AW, Scott AR, et al. Probability of fit failure with reuse of N95 mask respirators. Br J Anaesth 2020;125:e322-4.

10 CPAC Equipment I. Decontamination of $\mathrm{N} 95$ respirators using RapidHeat ${ }^{\mathrm{TM}} \mathrm{RH}-$ N95 high-velocity hot air technology, 2020. Available: https://www.cpac.com/ wp-content/uploads/2020/03/HVHA-N95-Mask-Decontaamination-Studies.pdf

$113 \mathrm{M} .9210 / 37021^{*}, 9211 / 37022^{*}$ particulate respirator N95 user instructions. multimedia3mcom 2007.

12 Centers for Disease Control and Prevention. Recommended guidance for extended use and limited reuse of N95 filtering Facepiece respirators in healthcare settings, 2020. Available: https://www.cdc.gov/niosh/topics/ hcwcontrols/recommendedguidanceextuse.html

13 Bergman MS, Viscusi DJ, Zhuang Z, et al. Impact of multiple consecutive donnings on filtering facepiece respirator fit. Am I Infect Control 2012;40:375-80

14 Meisenhelder C, Anderegg L, Preecha A. Effect of dry heat and autoclave decontamination cycles on N95 FFRs. medRxiv 2020.

15 Viscusi DJ, Bergman MS, Eimer BC, et al. Evaluation of five decontamination methods for filtering facepiece respirators. Ann Occup Hyg 2009;53:815-27.
16 Lin $\mathrm{T}-\mathrm{H}$, Chen $\mathrm{C}-\mathrm{C}$, Huang S-H, et al. Filter quality of electret masks in filtering 14.6-594 nm aerosol particles: effects of five decontamination methods. PLOS One 2017;12:e0186217.

17 Kumar A, Kasloff SB, Leung A. N95 mask decontamination using standard Hospital sterilization technologies. medRxiv 2020.

18 Yap TF, Liu Z, Shveda RA, et al. A predictive model of the temperaturedependent inactivation of coronaviruses. Appl Phys Lett 2020;117:060601.

19 Kampf G, Voss A, Scheithauer S. Inactivation of coronaviruses by heat. J Hosp Infect 2020;105:348-9.

20 Administration USFaD. Decontamination System EUAs for Personal Protective Equipment [web page], 2021. Available: https://www.fda.gov/medicaldevices/coronavirus-disease-2019-covid-19-emergency-use-authorizationsmedical-devices/decontamination-system-euas-personal-protectiveequipment

21 Services USDoHaH, Administration FaD, (CDRH) CfDaRH, (OPEQ) OoPEaQ. Enforcement policy for bioburden reduction systems using dry heat to support Single-User reuse of certain filtering Facepiece respirators during the coronavirus disease 2019 (COVID-19) public health emergency 2020

22 Science M. 3M ${ }^{T M}$ Particulate Respirator 9211/37022(AAD), N95 120/Case. Available: https://www.3m.com/3M/en_US/company-us/all-3m-products/ / 3M-Particulate-Respirator-9211-37022-AAD-N95-120-Case/?N=5002385+ 3294756845\&rt=rud

23 Grinshpun SA, Haruta H, Eninger RM, et al. Performance of an N95 filtering facepiece particulate respirator and a surgical mask during human breathing: two pathways for particle penetration. J Occup Environ Hyg 2009;6:593-603. 